\title{
Research on Application of PPP Pattern in Medical Treatment and Public Health of China
}

\author{
Yun Zhou \\ School of Literature, Law and Economics \\ Wuhan University of Science and Technology \\ Wuhan, Hubei, China 430081
}

\author{
Siyi Jiang \\ School of Literature, Law and Economics \\ Wuhan University of Science and Technology \\ Wuhan, Hubei, China 430081
}

\begin{abstract}
At present, public private partnership (PPP pattern) has been widely applied in the Medical Treatment and Public Health field of our country and achieved remarkable effects. This article, at first introduces the application background of PPP pattern in Medical Treatment and Public Health of our country, in the next briefly describes several common modes of PPP applying in the medical field of China in addition to issues discovered from the actual practices of those common PPP patterns followed with solutions raised separately directed against any discovered problems; at last it gives high expectations on the development of PPP pattern within Chinese Medical Treatment and Public Health industry.
\end{abstract}

Keywords-medical treatment and public health; public private partnership; social capital

\section{INTRODUCTION}

PPP refers to the English abbreviations of Public Private Partnership as usually interpreted, directing at the long-term cooperative relationship between public and private departments for benefit and risk sharing due to providing public products, mainly characterized in three aspects: on the basis of cooperation between governments and private departments, with the purpose of providing public products and capacity of realizing profit and risk sharing. In addition, the strengths of PPP lie in its contribution to relieving fiscal restraints from governments and separating risks, besides of benefits to economic development and social stability, in combination with abundant experience and examination through practicing in overseas medical and health filed, PPP, such an investment operation pattern gradually emerging in the recent years, is known as an efficient pattern of public private partnership worth of reference.

\section{APPLICATION BACKGROUND OF PPP PATTERN IN \\ MEDICAL TREATMENT AND PUBLIC HEALTH INDUSTRY}

As Chinese economy develops, leading to dramatic improvement of material standard of people living, the demands for diversified medical health care are constantly increasing as well and the population aging process is further fastened. As of the end of 2015, according to the data from national statistical yearbook, it is discovered that the population of Chinese people at and above the age of 60 reaches up to 222 million accounting for $16.1 \%$ of the gross population, and people at and above the age of 65 cover
143.86 million population and occupy $10.5 \%$. Thus, medical treatment and health filed has become one of the major domains for PPP pattern application. Some foreign data display that medical PPP project is most widely spread in British with a proportion up $49 \%$, nevertheless the medical PPP project of our country, by June of this year only develops under $6 \%$ of proportion pale by comparison with other countries, which also signifies great development space in the future.

Chinese government has unveiled many policies to create favorable environment for the development of PPP pattern in the medical field of our country. For example, in March 2005, the State Council issues Several Opinions on Encouraging, Supporting and Guiding the Development of Individual Private and Other Non-public Ownership Economy which as a guidance document, allows capital flowing into public utility, infrastructure and other domains. In May 2010, the State Council issues Several Opinions on Encouraging and Guiding the Construction Development of Private Investment putting forward "encouraging social capitals to actively involve the country's medical health career". In September 2014, the State Council suggests to standardize and rectify local governments to raise loans for financial institutions by PPP pattern, which offers positive supports for PPP development. In October 2014, the ministry of finance announces that debts in stock of regional governments will be attributed to clearance and discrimination methods of budget management to screen out the developing projects suitable for PPP application, which provides convenience for the PPP development. In December 2014, three documents on the cooperative mechanism of government and social capitals issued by the State Council, National Development and Reform Commission and ministry of finance come on to furnish space for the development and improvement of policies on medical field.

\section{PRACTICE OF PPP PATTERN IN CHINESE MEDICAL FIELD}

\section{A. Mutual Complementary Cooperation on Superior Resources - New Century International Children's Hospital}

The domestic first children's hospital with the international advanced level - New Century International Children's Hospital has developed first-rate medical experts and high- 
ranking nurse teams in combination with preeminent comprehensive health care service level, by which it has been dedicated to provide services for infants, school children and teenagers and gained public praises. In 2006, New Century International Children's Hospital realized the first cooperation with Beijing Children's Hospital, Capital Medical University, and cooperating with such a domestic excellent public children's hospital immensely satisfies multi-layered health demands of patients. In the past, since the large daily outpatient visits to Beijing Children's Hospital caused long waiting also great difficulty in timely meeting the medical demands of patients, the two hospitals chimed in easily and smoothly carried out the cooperation.

The operation is beneficial to both hospitals in many aspects, including realizing cooperation and mutual benefits, satisfying demands of multi-layered patients and above all, fulfilling complementary advantages.

Considering that some large-scale equipment such as MR and $\mathrm{CT}$ is only applicable for 5\% patients suffering extremely complex diseases and according to information data analysis, New Century International Children's Hospital suggests that some large scale equipment rarely useable should be narrowed down to reasonably reduce input and realize more efficient and maximized expenses and consumption of limited resources so as to provide more superior-quality services, thus the two hospitals decide to adopt the mutual complementary cooperation of superior resources: the former will purchase and use the medical facilities and services of the latter, while the doctors of latter are responsible for providing expert diagnosing services and conducting management. By this way, it can more than save a large amount of expenditure for directly purchasing expensive apparatuses of New Century International Children's Hospital and be in favor of creating opportunities for the doctors of Beijing Children's Hospital to learn management and accumulate more experience for providing high-quality and high-efficient as well as convenient and fast medical services of patients, which is definitely worth of reference.

\section{B. Trustee Mode - CR Phoenix}

Phoenix Healthcare Group, a relatively large privately operated medical treatment group of our country, mainly earns profits through the operation and management of hospitals and supply chains etc.

CR Phoenix has signed a framework contract for cooperative and joint construction with State Administration of Work Safety and CITIC Trust to establish the "Zhong Ankang Medical Industry Investment (Beijing) Co., Ltd." jointly operated and owned by the public and private, and the company will conduct mutual assistance and construction with China Meitan General Hospital and Shilong Hospital under the holdings of State Administration of Work Safety; in addition, CR Phoenix is successfully listed in Hong Kong in November 2013 and from the latter half of 2014, has published several cooperative projects and signed the contract for cooperative and joint construction with some hospitals of Beijing.

The typical pattern recently adopted by CR Phoenix is to complete collaborative and joint construction matters for companies to be founded; while the difference lies in the percentage of shareholdings to $\mathrm{CR}$ Phoenix. The registered capital of Zhong Ankang Company is targeted on 1 billion yuan, including $35 \%$ for CR Phoenix, $40 \%$ for State Administration of Work Safety and 25\% for CITIC Trust, and the State Administration of Work Safety will apply $90 \%$ of total assets of China Meitan General Hospital to realize the investment on the joint venture, while the other parties will implement shareholdings by cash contribution.

Another joint venture Beijing Jingmei Group General Hospital, for short JMH, is established jointly by CR Phoenix with $70 \%$ of equity and Jingmei Group with $30 \%$ of equity.

Under the contract concluded with Baoding city of Hebei province, CR Phoenix will found a wholly-owned subsidiary corporation in Baoding with a full-amount registered capital provided and by the year of 2021 , will realize 0.3 billion USD of total investment on the subsidiary.

The first mission for Zhong Ankang is to complete the capital reorganization and cooperative construction of China Meitan General Hospital and Shilong Hospital, along with the long-term objective on establishing and forming the network system integrating "medical treatment, health care and disease prevention", and Jingmei is responsible for conducting general reform on Jingmei Group General Hospital.

Baoding Phoenix commonly utilizes the public and private cooperative mode to achieve successful and fruitful cooperation with public medical institutions and medical educational institutions and further to bring medical treatment projects under key propulsive goals into the cooperation scope. Besides, that stipulated by the contract is to put the experimentation on demonstrated hospitals of Baoding in a core position for public fulfilling hospital reforms during the initial period of cooperative construction and then to enlarge the scope of cooperation henceforth.

CR Phoenix has seized the reform opportunity and participated in the public hospital reform of Mentougou District Hospital, on the grounds of which, it was smoothly listed in Hong Kong in November 2013 and from the second half of 2014, published some of relevant cooperative projects and concluded IOT trusteeship agreements with many hospitals in Beijing. Under such trusteeship, CR Phoenix will input corresponding medical facilities and equipment to improve the medical service level and ability of the hospital and to exchange rights and interests according to performance on management and operation of relevant hospitals within 19 to 48 years. Some of those hospitals should regular repay the input capitals from Phoenix Healthcare Group.

As of the midterm of 2014, Phoenix Healthcare Group has developed ten big comprehensive hospitals, one medium size hospital and twenty-eight community clinics in the list of managed medical service institutions.

The trusteeship management mode is noticeably characterized in not shaking the proprietorship root of hospitals, and under which the investors can only acquire handling expense from hospitals by taking advantage of entrusted operation and management profits of supply chains without changing property relations of hospitals. 


\section{Shareholding Reform Mode - Kunming Children's Hospital}

In April 2012, Kunming Children's Hospital successfully implemented the public-private partnership pattern, and CR Healthcare and Kunming municipal sanitary bureau reached cooperation intention to participate in the foundation of $\mathrm{CR}$ Kunming Children's Hospital and jointly conduct management. Such a new reform mode gives more liberty and decisionmaking power to the original public hospitals and simultaneously improves wage and treatment of medical personnel, in addition to perfecting income distribution mechanism and accelerating the improvement of service level.

\section{PRoblems of the DeVelopment of PPP in Chinese MEDICAL FIELD TO BE SOLVED}

Confronted with the new directions and fields, opportunities enjoy simultaneous existence with challenges and risks. Problems remain in dire need of solutions on the current application and development of PPP in the medical field of our country.

Firstly, an obstacle to positively promote the public and private cooperative pattern in the medical field of our country at the present stage results from excessively centralized solidified medical resource allocations instead of capital issues. As survey goes, a multitude of dominated talents are monopolized by public top three hospitals, which, on the basis of supply and demand consistency principle, will result in the current phenomenon with severely crowded patients in large public hospitals leaving all those small and medium size hospitals in a deserted embarrassment, which vividly shows the Matthew Effect in medical domain. Therefore, some of small and medium size hospitals have to be urgently drawn into the wave of social capital reorganization; however, adopting PPP pattern can but quickly solving the problem of capital shortage, and is incapable of timely redeploying highlevel medical talents, so the deficiency of manpower resources is an outstanding issue. Furthermore, it cannot orderly connect and impel chains of management and is difficult to quickly improve the operation efficiency.

So far, to solve the problem of solidified and hardly flowing talents, the national and regional governments have launched the policy "multi-point practice medicine" in succession, but for thoroughly activating the medical talent markets, plenty of tests are waiting. The main reason is that the implementation of the policy "multi-point practice medicine" touches upon multiple departments which are currently in an impeded connection and indistinct division of duties, straightly blocking the reform progress. In case the progress of practice medicine policy diverges from the reform of personnel system, doctors who are under the authorized strengths of public institutions will be imposed restrictions on conferring of academic titles and acquiring social security and welfare etc. by practice place of public institutions. In addition, no law or policy has ever expressly stipulated the responsibility for medical risks shared by doctors under "multi-point practice medicine" policy; thus, provided that any medical negligence occurs, it will easily lead to ambiguity and confusion of power and duties. Precisely because of those issues above, most doctors have had no confidence in the polity yet and held a wait-and-see attitude. The policy cannot actually activate the talent resources of medical talent market until completely breaking down all obstacles of current policies and schemes, synchronously promoting reforms of systems about continuous education, remuneration, assessment, welfare and social security etc. to fundamentally dispel the doubts of hospitals and doctors and get through the last barrier, so that more patients can enjoy more superior and abundant medical resources and hospitals operate more efficiently.

Secondly, shortage of coordinative mechanisms for PPP pattern has caused many barriers to supervision and administration and also enormously increased expenditures and difficulties in the involvement and attendance of social capitals in medical system reform. Medical treatment and public health is closely bound up with daily life of everyone, and in turn, people are quite unimaginably sensitive to both price and quality of services provided by hospitals, therefore, which requires governments to formulate a series of specialized laws and regulatory framework to systematically and comprehensively specify various links, regulatory mechanism, dispute settlement mechanism and the final handover mechanism concerning all aspects of medical PPP projects. At present, the mode adopted by our country, with regard to PPP, only embodies on "policies" made upon "notices" issued by ministries and commissions, with an inferior force of law. Moreover, each competent authority can no more than make decisions from their own point of view and under the separation between laws and regulations, which causes insufficient connections and incomprehensive polices; considering that, the governments must give a necessary and reasonable interpretation and explanation on the currently characteristic stockholding system non-profit pattern and standardize the return paths for private capitals involved in PPP projects by now, as well as stipulate the legitimate purposes of any earnings for public authorities and establish compliant model agreements and project process specifications before the final conditional stability for carrying out legislative regulations oriented to public hospitals.

Thirdly, the unclear rights and responsibilities and unknown demarcation exist between the market and governments. In such case, governments are not only stakeholders but the project supervisor; in spite of normative contracts concluded by enterprises to detailedly restrain the rights, obligations and responsibilities of all parties in connection to cooperative projects, the governments are still exerting mighty powers upon an inviolably and greatly authoritative status during the investment and operation of some projects; in case of any violations of governments, significant impacts will be brought to financing costs and project earnings of investors and prejudice the progresses of other correlative projects. Furthermore, medical field manifests itself as a capital intensive appearance by which it will cost large quantities of time and funds for preliminary scheming and post-stage operation; nevertheless, the current problem lies in insufficiently perfect and considerate feasibility and estimated efficacy of several cooperative projects on medical public private partnership, for instance, according to the plan, the authorities concerned intend to attract and absorb more 
private investment and capital input and promise to provide a certain range of capital guarantee for operators of projects; however, once any crises occur during the business operation, the authorities fail to timely perform the relevant regulations, and simultaneously no such a complete and sound supervisory mechanism or system has been ever formulated to restrain government behaviors, which, indeed, creates lots of obstacles for the practice of projects. On the other hand, by the rights entitled or empowered to the governments to exercise public powers on behalf of citizens, it is more likely to linking purposes and interests of governments with private units, not have to mention that disadvantage enterprise are all at, during the weigh and balance on respective rights and obligations, without operative constraints on rights by laws and contracts, enterprises have to frequently undertake extra risks and losses. In conclusion, the nation should attach more importance to the supervision and governance of public-private joint-operated projects and assign and practice the responsibilities to designate departments. Moreover, characterized in long time, large expenditure, wide span from finance, law to construction etc., the public-private joint-operated projects require the proper authorities to be provided with high ability to negotiation and governance and with professionals and experts. Eventually after the provisions on dealing with behaviors of breaching promises of public authorities are defined and clarified, it also requires determining the risks and costs (such as price policies and insurance payment risks) during the longterm medical service cooperation.

At last, since contradictions exist between the public welfare and profit pursuit of social capitals after hospital system transformation, it is necessary for us to, during the presentence of PPP pattern in hospital reforms, incorporate the five aspects including government, management level, staff, hospital and investor into consideration and for relieving the relevant conflicts and under the premise of non-profit cooperation, the private capitals can acquire the corresponding earnings and returns through the management of logistics and property; moreover, debt financing, even if as one of the channels of private capital relative gains, is out of circulation with any concrete business activities. In addition, government agencies are required to be strict accordance with and execute the term of cooperation and withdrawal mechanism as regulated before retreating during the hospital operation and services in sound conditions on the basis of full understanding on the public welfare nature.

\section{CONCLUSION}

It is a significant mode that to utilize public private partnership in the medical field to improve efficiency of supply and quality of public health services, so in the future, we are still required to further provide corresponding and matching supports and promote the efficient utilization of public health services to acquire long-term development of PPP pattern in medical field.

\section{REFERENCES}

[1] Yang Lei. Application of PPP Pattern in Medical Treatment and Public Health Industry[J]. Management \& Technolosy of SME, 2015, (7): 161162.

[2] Chen Yulan. Research on the Application of PPP Pattern of Medical Field[J]. Market Forum, 2016, (4):25-27.

[3] Mao Qingxiang, Pan Gao. Application of PPP Pattern of Medical Treatment and Public Health Industry[J]. CO-Oerativeconomy \& Science, 2012, (9):34-35.

[4] Li Lu, Sun Zhangxue, Zhang Luqin. Problems and Suggestions on the PPP Promotion in Current Medical Treatment, 2016, (6):39-41.

[5] E.S. Sarwar, translated by Zhou Zhiren etc. Privatization and PPP Pattern[M]. Beijing: China Renmin University Press, 2014.

[6] Xie Jingyu. Application of PPP Pattern in the Public Hospital Reform[J]. Business Accounting, 2016, (14):80-82.

[7] Kang Jingning. Research on the Application of PPP Pattern in Medical Treatment and Public Health Field in China - Concurrent Discuss on Functions of PPP Pattern in Transformation of Government Public Administration [J]. Straits Science, 2013, (10):3-4 